\title{
EDITORIAL
}

\section{DECLARACIÓN DE LA HABANA \\ HACIA EL ACCESO EQUITATIVO A LA INFORMACIÓN EN SALUD}

\begin{abstract}
Nosotros, los participantes en la Segunda Reunión de Coordinación Regional de la Biblioteca Virtual en Salud (BVS) y en el V Congreso Regional de Información en Ciencias de la Salud (V-CRICS) de América Latina y el Caribe, reunidos en La Habana, Cuba, del 23 al 27 de Abril de 2001,
\end{abstract}

\section{CONSIDERANDO}

que la información científico técnica en salud es un bien público esencial para el desarrollo social, cuya diseminación universal y equitativa debe ser asegurada por políticas públicas nacionales e internacionales;

que las desigualdades injustas, innecesarias y evitables en las condiciones de salud de los individuos y grupos de la población son consecuencia de las diferencias de recursos y oportunidades, destacándose el acceso desigual a las fuentes de información en salud;

que cada vez más el combate contra las inequidades y la pobreza pasa por el fortalecimiento de la capacidad de participación política y social de los excluidos, a través del acceso a la información y el conocimiento; que la Biblioteca Virtual en Salud es un poderoso instrumento para democratizar la información y el conocimiento;

\section{REITERAMOS}

nuestro compromiso con la construcción de la BVS como espacio de cooperación entre países, para fortalecer la capacidad de aprovechar las oportunidades que ofrecen las nuevas tecnologías de información y comunicación en pro de la equidad en salud;

\section{INSTAMOS}

a los gobiernos de los países de las Américas a que individual y colectivamente definan políticas públicas de acceso equitativo a la información de calidad para promover el alcance por todos de los derechos sociales básicos;

\section{CONVOCAMOS}

a los organismos públicos y privados, nacionales e internacionales, comprometidos con el bienestar y la salud de los pueblos de América Latina y el Caribe a unir esfuerzos en la construcción de la Biblioteca Virtual en Salud, para que todos los ciudadanos tengan acceso a la información y al conocimiento necesarios a la construcción de su salud individual y colectiva. 